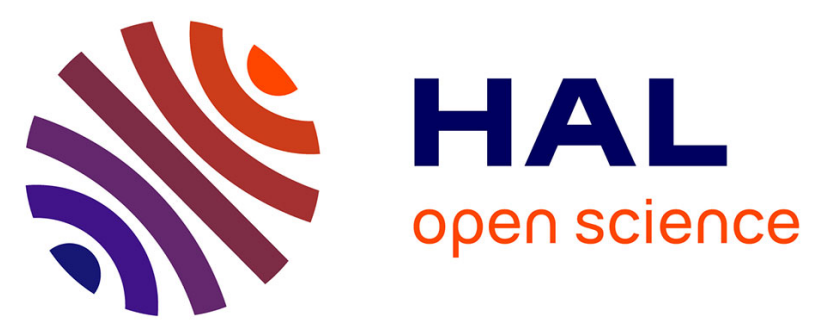

\title{
Internal Charging Analysis of a Space Instrument in PEO With a Dedicated Modeling Chain
}

Benjamin Jeanty-Ruard, Pierre Sarrailh, Denis Payan, Arnaud Trouche, Nicolas Chabalier, Amandine Champlain, Giovanni Santin, Julien Forest

\section{To cite this version:}

Benjamin Jeanty-Ruard, Pierre Sarrailh, Denis Payan, Arnaud Trouche, Nicolas Chabalier, et al.. Internal Charging Analysis of a Space Instrument in PEO With a Dedicated Modeling Chain. IEEE Transactions on Plasma Science, 2019, 47 (8), pp.3699-3709. 10.1109/TPS.2019.2911408 . hal02507375

\section{HAL Id: hal-02507375 \\ https://hal.science/hal-02507375}

Submitted on 2 Nov 2021

HAL is a multi-disciplinary open access archive for the deposit and dissemination of scientific research documents, whether they are published or not. The documents may come from teaching and research institutions in France or abroad, or from public or private research centers.
L'archive ouverte pluridisciplinaire HAL, est destinée au dépôt et à la diffusion de documents scientifiques de niveau recherche, publiés ou non, émanant des établissements d'enseignement et de recherche français ou étrangers, des laboratoires publics ou privés. 


\title{
Internal Charging Analysis of a Space Instrument in PEO With a Dedicated Modeling Chain
}

\author{
B. Jeanty-Ruard ${ }^{\circledR}$, P. Sarrailh ${ }^{\circledR}$, D. Payan ${ }^{\circledR}$, A. Trouche, \\ N. Chabalier, A. Champlain, G. Santin ${ }^{\circledR}$, and J. Forest
}

\begin{abstract}
An internal charging (IC) analysis of the active measurement box of electrostatic risk (AMBER) instrument onboard Jason-3 in polar earth orbit (PEO) has been performed with a new dedicated modeling chain. The ExtendeD Gdml Editor (EDGE) application is in charge of the definition of the computer-aided design (CAD) model. The Modelling of Radiation (MoORa) front end helps to configure, control, and postprocess the ESA/GRAS Monte Carlo simulation based on Geant4 to model the transport of space radiation environment. Finally, using the radiation results, the IC analysis, including internal electric fields and risk of discharge, is performed with the Spacecraft Plasma Interaction Software (SPIS)-IC 3-D time-dependent simulation code.
\end{abstract}

Index Terms-Active measurement box of electrostatic risk (AMBER) instrument, charge, computer-aided design (CAD), dose, ExtendeD Gdml Editor (EDGE), Geant4, GRAS, internal charging (IC), Jason-3, modeling chain, Modelling Of Radiation (MoORa), Monte Carlo, multiscale, radiation, simulation, Spacecraft Plasma Interaction Software (SPIS)-IC.

\section{INTRODUCTION}

D UE to the technological evolutions and the new mission profiles, the challenge of studying Internal Charging (IC) effects is an increasing issue for space systems. It may potentially impact all thick dielectric or ungrounded metallic parts inside the spacecraft. Several kinds of equipment can be affected like scientific instruments that are outside the spacecraft and thus especially exposed to the radiation from the environment. As explained in [1], the shielding has an impact on the IC analysis. It has been demonstrated that the global geometry system must, therefore, be taken into account to be able to precisely assess the IC risk. Due to the lack of in-flight feedbacks, the integration of sensitive components into space applications requires both accurate Modelling Of Radiation (MoOra) and IC analysis, as well as new simulation tools.

B. Jeanty-Ruard, A. Trouche, N. Chabalier, and A. Champlain are with Artenum Toulouse, 31520 Ramonville Saint Agne, France (e-mail: ruard@artenum.com).

P. Sarrailh is with ONERA, 31000 Toulouse, France.

D. Payan is with CNES, 31400 Toulouse, France.

G. Santin is with ESA, 2201 AZ Noordwijk, The Netherlands.

J. Forest is with Artenum Paris, 75010 Paris, France.

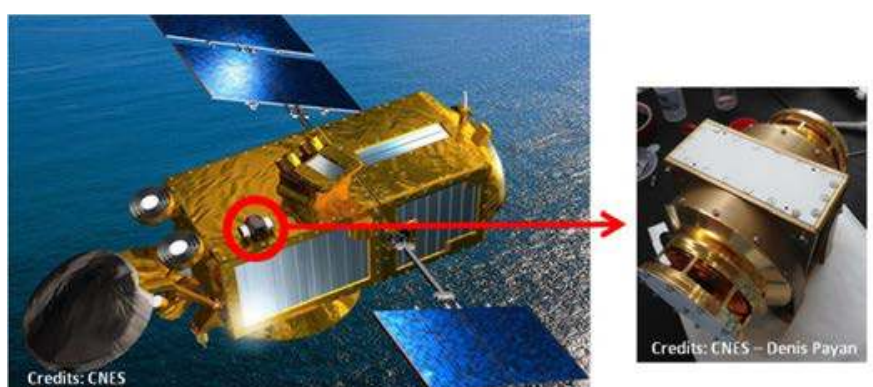

Fig. 1. AMBER instrument on Jason-3 mission

The active measurement box of electrostatic risk (AMBER) instrument [2], [3] developed at CNES aims to measure the spacecraft electric potential and auroral particle precipitations for electrons and ions in the energy range of $10 \mathrm{eV}-28 \mathrm{keV}$. AMBER is currently onboard the ocean topography mapper spacecraft Jason-3, as illustrated in Fig. 1. Jason-3 is in a circular nonsun-synchronously orbit; $1336-\mathrm{km}$ altitude, $66.038^{\circ}$ inclination [4].

Even if the current integration on Jason-3 is not predicted to present any particular risk in terms of IC (i.e., the transit duration in the polar zone is too short), it can be used as a good practical case for multiscale modeling of IC. A step-by-step methodology is presented in this paper to analyze the IC effects on a space system.

In Section II, a modeling chain able to perform such analysis is presented. In Section III, the radiation transport analysis of the AMBER instrument, needed as input to model accurately the IC phenomenon, is detailed. In Section IV, the IC analysis on the AMBER instrument is performed and the results are analyzed. Finally, in Section V, the modeling chain is applied on the AMBER instrument with the Jason-3 body and the results are discussed.

\section{Presentation of the Modeling Chain for INTERNAL CHARGING STUDY}

The proposed modeling chain used for IC analysis is composed of several steps, as described in Fig. 2, only partially handled, or through different tools, in previous IC works.

To evaluate the IC risk, 1-D modeling tools, like DICTAT [5] or NUMIT 2 [6], have been widely used. However, these models imply to reduce the studied systems to a one-dimension layer approximation, which is not relevant 


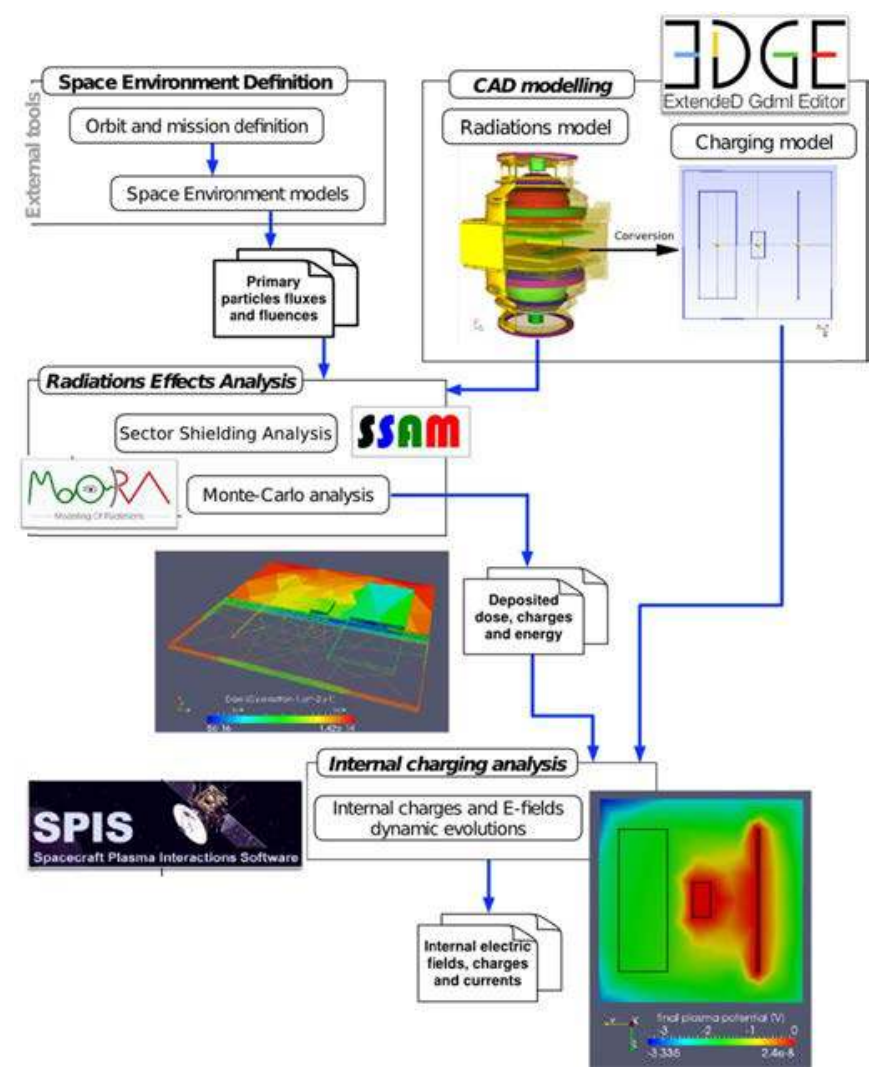

Fig. 2. IC modeling chain.

for most realistic systems. Moreover, these tools estimate the dose and charge deposition rates through analytical models or by fitting precalculated profiles, constituting another strong approximation, especially for electrons. Spacecraft Plasma Interaction Software (SPIS)-IC [7] was the first answer to address the 3-D dynamic evolutions of charges in dielectrics, but the primary charges and dose depositions remained delegated to external tools. The ELSHIELD [8], [9] and CIRSOS [10] ESA projects (e.g.,) were a first exploration of the possibilities to interface SPIS-IC with 3-D Monte Carlo particle transport. The work has been mainly focused on the conversion bridges for exchanged data and to identify usage scenario. The 3-D NUMIT is a recent refactoring of NUMIT [11] following a similar approach and running 3-D finite-element-based model to solve the slow conduction dynamics.

Most of these modeling chains remain not integrated or based on different external tools and/or requiring a high level of expertise in both radiation transport and charging. As discussed in the following sections, a multiphysics/multimodel approach requires an adaptation of the modeled system (e.g., geometry and material properties) for each step of the chain. To address these issues, the modeling chain presented in this paper has been built following a step-by-step methodology and including additional pre- and postprocessing tools, such as a dedicated computer-aided design (CAD) editor, conversions facilities, embedded mesher, and rich 2-D/3-D postprocessing functions. Last, the unstructured meshing for the charging model allows processing very complex shapes, including strong anisotropies, like electronics subsystems.

\section{A. Analysis of Effects of Radiation Transport}

The basis of radiation transport analysis for IC is to be able to compute the dose deposition rate and the charge implantation rate inside the sensitive components of the spacecraft. Charge and dose deposition inside components in the considered case of AMBER is mainly due to high-energy electrons $(\sim \mathrm{MeV})$ from the external environmental fluxes. As we will show below, the sensitive components are not directly exposed to the plasma from the environment; thus, the influence from the shielding should be taken into account carefully. The transport of such particles through the spacecraft materials can be performed, for instance with Monte Carlo codes which model all particle interactions (secondary electron emission, Bremsstrahlung, ...). When these primary particles and their secondary particles are interacting with the matter, the particles are losing a fraction or all its energy that induces dose rate and a charge deposition rate. The dose rate creates the radiation-induced conductivity (RIC) [7] of materials that mitigates the charge implantation in the material. The dynamic evolution of electric potential is due to the equilibrium between both phenomena: the implantation that increases the charge number in the dielectric and the conductivity that permits to the charges to flow to the metallic part of the components. These phenomena influence the probability of electrostatic discharges occurring inside the dielectrics. A radiation transport analysis computing the dose and charge deposition rates should, therefore, be performed prior to the IC analysis. In the case studied here, the materials used are not sensitive in term of aging to such low dose accumulation. Thus, the aging due to radiation is not taken into account. Fig. 2 presents the whole modeling chain used to model the IC risk.

The configuration of radiation transport analysis needs several input parameters illustrated in different blocks in Fig. 2 and described below.

1) CAD Modeling: The calculation of the dose and charge deposition rates is very sensitive to the shielding configuration, in terms of shape, thickness, and material [7]. It is, therefore, necessary to define precise and realistic geometries for transport models. The Geant4 library, able to consider realistic 3-D systems, including accurate physics models, has become over time a major reference for modeling of particle-matter interactions. It is the foundation of most current radiation transport simulation tools, based on direct or reverse Monte Carlo models. In Geant4-based tools, the description of the geometry is carried out either by internally coding $\mathrm{C}++$ classes or via an external geometry description, such as through XML files in GDML format [12], [13], for which the number of available CAD editor tools is very limited, until now. In this paper, the new solution ExtendeD Gdml Editor (EDGE) [14] has been used for CAD modeling.

EDGE is a user-friendly application able to load and edit GDML geometries, including real-time 3-D visualization. The tool enables users to create and edit the GDML files necessary for the radiation transport analysis, including the material description of the geometrical system.

2) Space Environment Definition: The primary incoming particle fluxes or fluences, in both species and 
energy spectrum, is an input to the radiation transport effects modeling, and depend on the space environment crossed and on the orbit profile. Today, numerous validated models and in-flight data are available, including online services like ESA/SPENVIS, and the produced spectra are compliant with the present chain.

3) Radiation Transport Effects Analysis: There are two major approaches to model the radiation transport effects on devices.

a) Sector shielding analysis: In an engineering context, the sector shielding analysis is a basic approach usually used to evaluate the dose on a device. This approach is based on a geometric ray-tracing method to compute the equivalent shielding in each direction around one specific point. Combined with a dose depth curve, it is then possible to compute the dose in a specific point. Such an approach presents the major advantage to be simple and fast. In the present modeling chain (see Fig. 2), the sector shielding analysis approach is addressed by tools like Sector Shielding Analysis Module (SSAM), available inside EDGE.

However, depending on the application cases, the sector shielding analysis may not be accurate enough, especially for an IC analysis downstream. Most of the time, inside the radiation belts (e.g., auroral regions), the IC phenomenon is dominated by the energetic electrons. The protons and heavier particle can be neglected. Electrons trajectory inside matter cannot be considered as straight lines as the scattering collisions are significant. Simple ray-tracing analyses cannot be considered as more than a preanalysis to study the global system. Indeed, the geometry is then approximated by sectored spheres where all materials are converted into an equivalent thickness of aluminum. Electron trajectories are different between the real geometry of the spacecraft and its equivalent one computed by ray tracing. Even if precomputed dose depth curves include all the scattering, particles which may come from another sector are neglected. In addition, IC models need to take into account the distribution of dose rate and charge deposition rate over the whole sensitive volumes, not only in one point. In this case, all simulation time gains of the ray-tracing technique are lost by the necessity to rerun them numerous times to completely cover the targeted component.

b) Monte Carlo: Alternatively, the 3-D Monte Carlo approach, where the particle trajectories are really computed, takes into account the interaction with the matter and more precisely addresses the physical processes leading to more accurate results compared to sector shielding approach [15]. The direct Monte Carlo approach can be costly in terms of simulation time. However, both careful identification of sensitive components and good biasing techniques make such an approach fully compliant with the constraints of operational studies in an engineering context in terms of run duration.

Currently, the Geant 4 library is one of the reference tools to perform 3-D Monte Carlo radiation transport [16]. In the space domain, the open code ESA/GRAS, based on Geant4, has become a standard [17], [18] and, for this reason, has been integrated into the present chain. Until now, GRAS can only be used with command-line using a control script and lacks a GUI for nonexperts. In order to help in the use of GRAS,

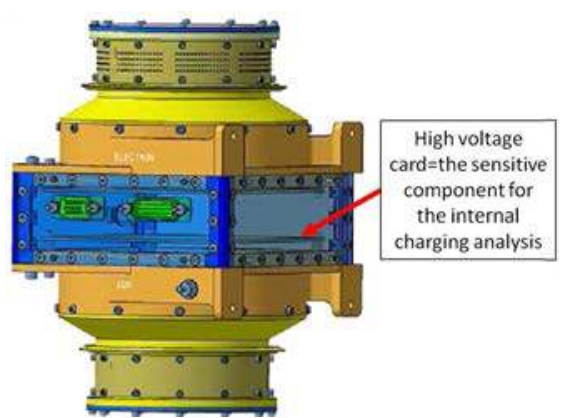

Fig. 3. AMBER instrument model in STEP AP file format, including all detailed elements.

the MoORa tool [19] has been used. It is a GUI application, able to set up GRAS inputs, monitor the simulation, and postprocess the results in 3-D and in $y=f(x)$ type plots.

In this modeling chain, the sector shielding analysis is used to validate the parameter inputs for Monte Carlo simulation and GRAS/MoORa is used to compute the dose and charge deposition rates with better physic results regarding the sector shielding analysis [15].

\section{B. Internal Charging Analysis}

After the radiation transport analysis, the last part of the modeling chain presented in Fig. 2 is the IC analysis. This second part uses as inputs the 3-D maps of the dose and charge deposition rates resulting from the radiation transport simulation. Since 2001, Spacecraft Plasma Interaction Software (SPIS) is widely used for surface charging analysis [20], [21]. Open-source and available through the SPINE community [22], SPIS is based on an electrostatic 3-D plasma model, called SPIS-NUM, and a volume unstructured mesh. Over the span of several projects, ESA has encouraged the development of new numerical models to compute IC effects. These efforts, added to ONERA's internal research and development activities, have recently led to a dedicated branch of the SPIS software, called SPIS-IC, for IC simulations. Detailed models and validation of the software have been presented in several publications [7], [23].

The presented modeling chain has been used to model the AMBER instrument to study the IC effects.

\section{RADiation TRANSPORT StUdy}

The MoORa/GRAS tools have been used to precisely model, in a realistic geometry, the locally dose and charge deposition rates inside a sensitive component. These results will be used as input for the IC analysis in Section IV.

\section{A. Geometrical Modeling for Radiation Transport Analysis}

As with most radiation transport compliance analysis, the initial geometric description of the system is issued from the mechanical design working with a STEP-AP 203/214 design file. As illustrated in Fig. 3, this file defines all the geometric details of the casing, and the exact dimensions of the cards used in AMBER. No detail regarding the electrical circuits of the cards is specified in this file. However, 


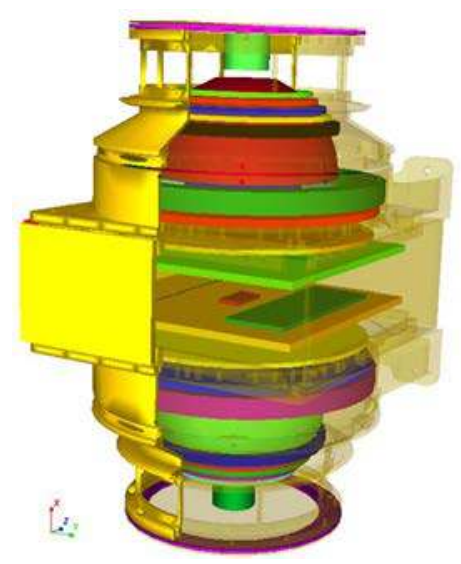

Fig. 4. AMBER STEP AP CAD file imported in EDGE and treated to be consistent with GRAS/Geant4 simulations.

this type of CAD model, BRep based and highly detailed, cannot be used as is for transport simulation. With EDGE, it is possible to convert this geometric description into a simplified and CSG-based CAD, compatible with GRAS/Geant4/MoORa tools.

As shown in Fig. 3, the STEP-AP file presents a lot of details that are irrelevant for radiation transport simulations, resulting in very long simulation time. The number of shapes defining the geometry, especially the primary faces (i.e., triangles) in tessellated shape, has a direct impact on the simulation time. For this reason, it is convenient to merge those small shapes, removing smaller components and filling the remaining holes.

Each shape of the input STEP-AP file is imported and converted into equivalent GDML native elements, with the option to ignore elements smaller than a given threshold volume. If most of these simplifications are done through the semiautomatized functions of EDGE, the resulting geometry must still be manually checked and may be edited to finalize this tailorization for radiation transport models. Fig. 4 shows the results of the geometry treatments as displayed in EDGE. In addition, EDGE might be used to check for the absence of overlaps between shapes of the geometry, ensuring a selfconsistent geometry, fully compliant with Geant4/GRAS or all other GEANT4-based codes.

In the first part of this paper, the Jason-3 hub and its relative shielding effect have been intentionally left out of the initial modeling. The modeling of the Jason-3 spacecraft and its impact on the results are taken into account and presented in Section V.

IC analysis is a multiscale process, but only some specific sensitive components, such as connectors [7], cables [23], or PCB cards, might be impacted and present charging issues. It is not relevant to study the whole system, for IC. In this paper, the analysis has been focused on the PCB high-voltage card inside AMBER.

In an IC analysis, one of the most critical aspects is the resulting electric field through the matter. This depends on the handled dielectric properties but also on the geometry of the studied element. In the configuration defined in this paper, in a PCB card, by its thin thickness, the induced electric field,

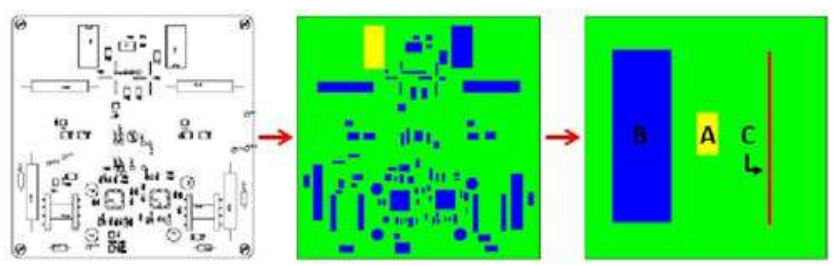

Fig. 5. Design and simplification of the electrical circuit of the high-voltage card.
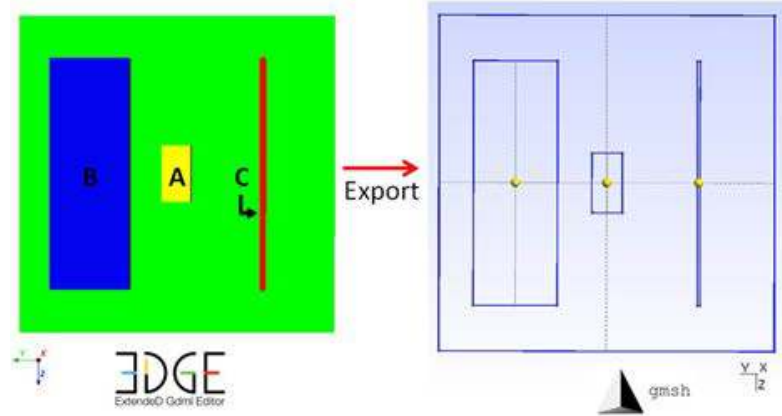

Fig. 6. Export of the high-voltage card from GDML (CAD for radiation transport simulation) to $\mathrm{Gmsh}$ format (CAD for IC simulation).

and risks of discharges or arcing, can be expected dominating through the thickness of the card (in this paper in the $x$-axis), rather than along its surface. In this case, the electric circuit of the PCB card does not need to be modeled with a high level of detail and can be simplified. The precise position of the components on the card is not relevant here then. As illustrated in Fig. 5, an equivalent circuit has been built using EDGE. To simplify the simulation, the components have been moved and reduced into a set of three main equivalent components. A field-programmable gate array (FPGA), called A and displayed in yellow on figures, has been kept as is, being a component of interest and relatively massive but centered on the top side of the card. Other electrical components of the circuit have been moved and merged into a single large equivalent component, called B and displayed in blue on figures. Last, the copper circuit has been reduced to a single strip, called C and displayed in red. The electrical circuit is electrically grounded.

As illustrated in Fig. 6, thanks to the export capabilities of EDGE, a Gmsh SPIS-compatible B-Rep equivalent model and a corresponding volume mesh file have been generated. This mesh aims to support the radiation transport results on the card and its components and transfer results in each tetrahedron to the IC model as an input.

A proper adaptation of the scoring mesh is important for the accuracy of the final results. It is critical to have a minimal description of the electrical field along the thickness of the card. This is why at least ten tetrahedra are necessary along the thickness of the card. In addition, due to the very small thickness of the card with respect to their other dimensions, an isotropic mesh will lead to a prohibitive number of elements. Conversely, in this paper, a thin meshing along the surface of the card is not necessary, so an anisotropic mesh has been specifically built with a higher refinement along the $\mathrm{x}$-axis. 
TABLE I

AMBER MATERIALS IN EDGE

\begin{tabular}{c|c|c} 
GDML shapes & Material type & Density $(\mathrm{g} / \mathrm{cm} 3)$ \\
\hline Casing & Aluminium & 2,7 \\
\hline Other internal components & Aluminium & 2,7 \\
\hline High voltage cards & Epoxy & 1,2 \\
\hline Components on high voltage card & Epoxy & 1,2 \\
\hline PCB cards & Epoxy & 1,2 \\
\hline Copper strip on the high tension card & Copper & 8,96 \\
\hline
\end{tabular}

TABLE II

INTEGRAL ELECTRON FLUX FOR MONTE CARLO GRAS SIMULATION

\begin{tabular}{|c|c|} 
Electron energy (MeV) & Flux $\left(\mathrm{cm}^{-2} \cdot \mathrm{s}^{-1} \cdot \mathrm{sr}^{-1}\right)$ \\
\hline 1,6 & 29500 \\
\hline 1,67 & 28500 \\
\hline 1,74 & 26000 \\
\hline 1,81 & 24500 \\
\hline 1,88 & 23000 \\
\hline 1,95 & 18500 \\
\hline 2,02 & 16000 \\
\hline 2,6 & 9500 \\
\hline
\end{tabular}

The materials assigned to the AMBER system are summarized in Table I. For the Monte Carlo simulation, the material properties used are fully defined by the density of the stoichiometric and isotopic compositions of materials. Thanks to EDGE, these materials have been built and assigned to the various shapes of AMBER.

\section{B. Space Environment Definition}

The Jason-2 and Jason-3 orbits are exactly the same with a polar earth orbit (PEO) to an altitude of $1336 \mathrm{~km}$ and $66.038^{\circ}$ of inclination The space environment considered in this paper is the same than Jason-2 orbit during three years and has been established in a previous work performed by the EREMS company [24] using a stationary environment model. The Jason-2 and Jason-3 orbits are exactly the same. The current analysis models the electrons coming from the space environment in the polar cusps. The protons could also be modeled, but their influence is considered negligible and not analyzed in this paper. Table II details electron flux coming from the environment used for the GRAS simulation. In this paper, the environment is considered constant in time. Charging mechanism is a dynamical process, potentially impacted by environmental events and time-varying environments; this can be handled in SPIS-IC [25].

Input parameters for the radiation transport analysis (i.e., energy spectrum, the geometry of space environment sources, and angular distribution) are fully defined through the MoORa GUI for GRAS, as illustrated in Fig. 7. The source geometry used for the GRAS simulation is the surface of a sphere with a radius of $13 \mathrm{~cm}$. The electrons are launched inward the sphere and with a cosine law angular distribution to reproduce an isotropic flux in the whole computational domain [26].

To reduce the simulation time of the direct Monte Carlo analysis, the input particle spectrum has been biased to only consider incoming electrons with enough energy to pass through the AMBER casing. A sector shielding analysis has been performed by the SSAM module of EDGE to determine

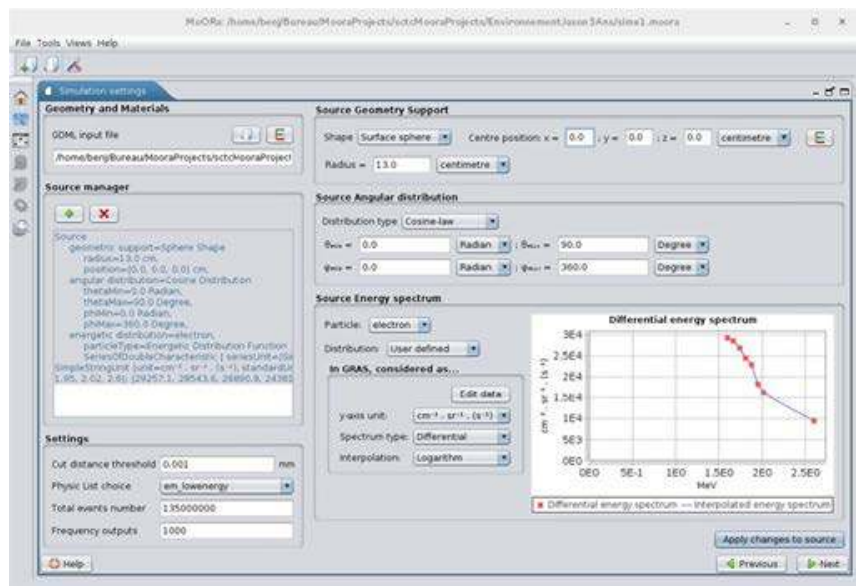

Fig. 7. Definition of the space environment in MoORa.

the mean thickness of equivalent aluminum in each direction around the studied card. This has been performed by the SSAM module of EDGE. Considering all directions, the minimum shielding value is $2.5 \mathrm{~mm}$, which is the same result than computed with other Sector Shielding Analysis codes [27]. Considering the electron range in aluminum, most electrons with energy lower than $1.6 \mathrm{MeV}$ are stopped by a few millimeters of aluminum and these particles never reach the target. According to this, a biased energy spectrum with no values below 1.6 MeV has been considered for the environmental particle source.

\section{GRAS Physical List Choice}

Numerous physical processes, such as scattering, collision, Bremsstrahlung, or nuclear interactions, are handled by various physic lists provided with Geant 4 . The selection of the most relevant physics list depends on the target modeling and may deeply impact the simulation cost. In this paper, the "em_standard_opt4" list has been selected for the GRAS simulations. This physics list is designed for application requiring higher accuracy of the electron, hadron, and ion tracking [28], [29]. The processes modeled by this physics list for electrons are ionization, Coulomb scattering, Bremsstrahlung, secondary emissions, and Positron annihilation. For the nonexpert users, the selection of the physics list is highly simplified and easier through the MoORa GUI.

More generally, MoORa allows the user to fully define and control the GRAS simulation in a simple What You See Is What You Get (WYSIWYG) interface. On the other hand, a rich text editor, with syntax coloring, lets advanced users edit input .mac script control files for GRAS and adapt them to specific cases. Live monitoring helps control the evolution of the simulation.

\section{Results}

For the same simulation (i.e., same geometry, material assignment, physic model, environment, and input parameters), GRAS is able to compute different results like the cumulated dose, averaged on given components in their 
TABLE III

Cumulative Dose by Components Computed By GRAS

\begin{tabular}{|l|c|}
\hline \multicolumn{2}{|c|}{ GRAS results } \\
\hline components & dose (kRad) \\
\hline high voltage card & 5,7 \\
\hline copper string & 9,7 \\
\hline single component & 4,4 \\
\hline concatenation of component & 4,7 \\
\hline
\end{tabular}

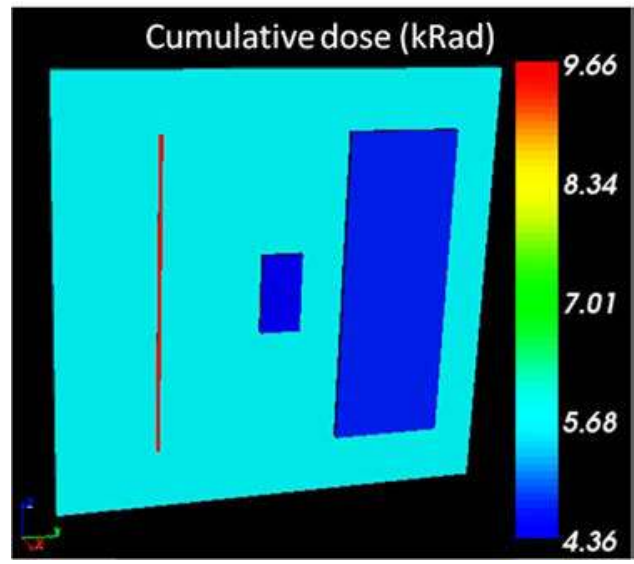

Fig. 8. Average of the cumulative dose computed by GRAS Monte Carlo simulation in various equivalent components over the high-voltage card.

wholes or the dose and charge deposition rates computed locally on each cell of a tetrahedral volume Gmsh mesh, discretizing the sensitive volumes. Both can be postprocessed and visualized in 3-D, directly in MoOra or exported to VTK files.

This is the locally scored dose and charge deposition rates which are relevant for IC analysis, as explained in Section II-A. However, to validate the settings of GRAS for both different results, the computation of the cumulative dose, individually averaged over the PCB card and the A, B, C elements, is calculated and compared with results of other tools and models.

1) Average of the Cumulative Dose Over Whole Components: The cumulative dose is computed for a total duration of three years in the space environment, described in Section III-B. The cumulative doses have been computed with several approaches, a direct Monte Carlo model with the GRAS/MoORa couple, on the one hand, and a sector shielding analysis, in the other hand, with, first, the SSAM of EDGE and, last, the legacy tool FASTRAD.

Table III gives the cumulated doses computed with GRAS/MoOra for each studied component. Due to its higher atomic number and density, the copper strip (C) logically gets the highest dose. Other components, i.e., the PCB card, the merged of elements (B) and the FPGA (A), present similar values between 4.4 and $5.7 \mathrm{kRad}$ for nonmetallic components, as illustrated also in Fig. 8. In comparison, Table IV presents the cumulative doses for equivalent components computed using two sector shielding analysis tools, EDGE/SSAM and Fastrad in another study [27], with the same environment and duration. These results are similar to those computed by GRAS, so we can conclude the settings of the GRAS
TABLE IV

Cumulative Dose Computed By Sector Shielding Analysis

\begin{tabular}{|c|c|c|c|}
\hline \multicolumn{4}{|c|}{ cumulative dose (kRad) with Sector Shielding analysis } \\
\hline \multicolumn{2}{|c|}{ Fastrad results } & \multicolumn{2}{|c|}{ SSAM results } \\
\hline min value & max value & min value & max value \\
\hline 5,2 & 6,4 & 6,1 & 7,4 \\
\hline
\end{tabular}

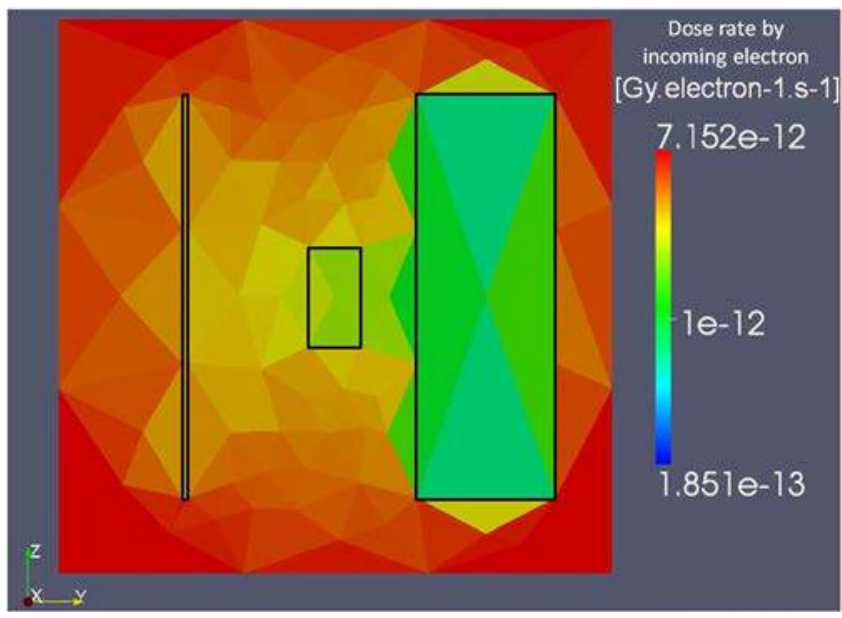

Fig. 9. Top view of the scoring of the dose rate by incoming electron computed by GRAS over the high-voltage card and its components.

simulation are correct and the distributed dose and charge deposition rates results, used for the IC simulation, computed in the same simulation are also correctly set for this paper.

The integration of a sector analysis module, like SSAM, in the present chain answers another practical issue with the possibility to measure on realistic geometries the equivalent thickness of aluminum in each direction and also the minimum, mean, and maximum shielding thicknesses view by the card. They are key input information for numerous 1-D IC models like used in DICTAT [30] as well as its possible anisotropy. In the case of the studied card and AMBER casing, the analysis done with SSAM gives a minimum shielding of $2.5 \mathrm{~mm}$, for a mean value about $12 \mathrm{~mm}$ and a maximum thickness of $25.7 \mathrm{~mm}$.

2) Distributed Dose and Charge Deposition Rates: The IC simulation needs distribution details regarding the dose and the charge deposition rates. Dose rate is computed for each tetrahedron in the meshed volume corresponding to the card and its components, as shown in Figs. 9 and 10. To ease the visualization of data, the mesh has been artificially inflated along the thickness axis in Fig. 10. Dose rate is computed when a particle loses energy in a tetrahedron. This result confirms that the dose rate is mainly deposited in the first micrometers of the card and corners, where the influence of self-shielding is less evident. Moreover, no major anisotropy is observed. This is consistent with the fact that the primary source has been defined as isotropic. In the present case, the relative smoothness of the dose rate in different tetrahedra seems to confirm that, with $10^{9}$ primary events, we have reached a statistic good enough for later charging analysis.

Figs. 11 and 12 show the corresponding absolute value of the charge deposition rate. As for the dose rate, the charge deposition rate is mainly concentrated in the first micrometers 


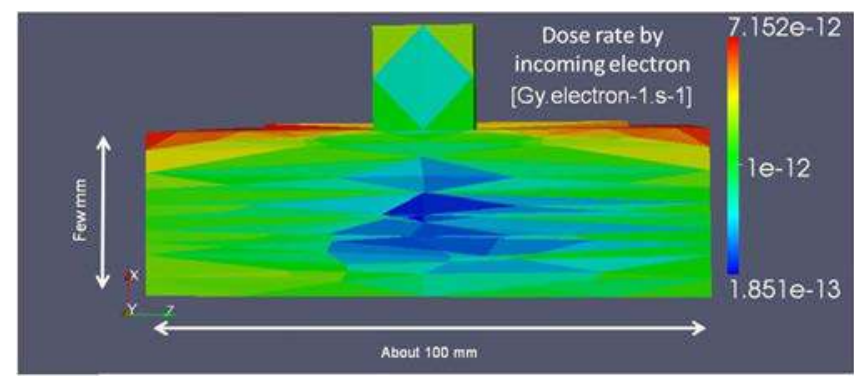

Fig. 10. Clipping of the dose rate by incoming electron computed by GRAS. View scaled along the thickness direction.

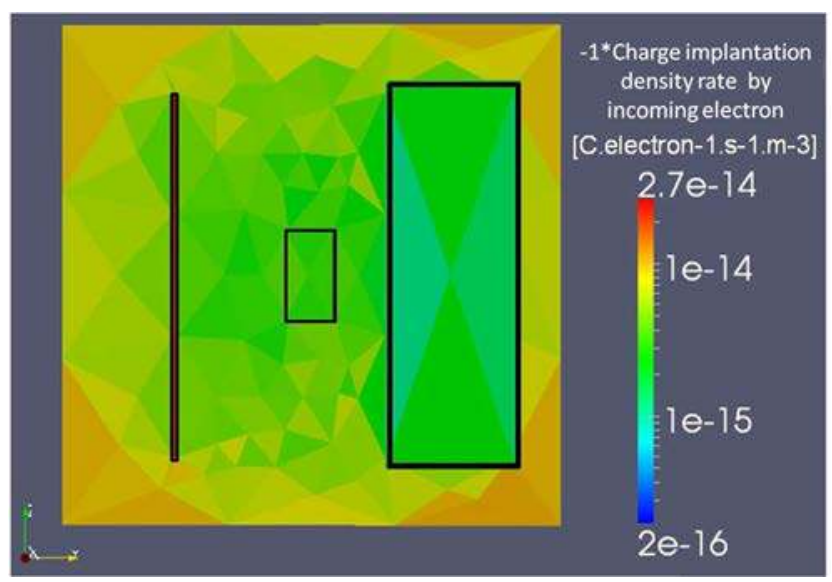

Fig. 11. Top view of the scoring of the implantation density rate density by incoming electron at the GRAS simulation surface boundary computed by GRAS over the high-voltage card and its components.

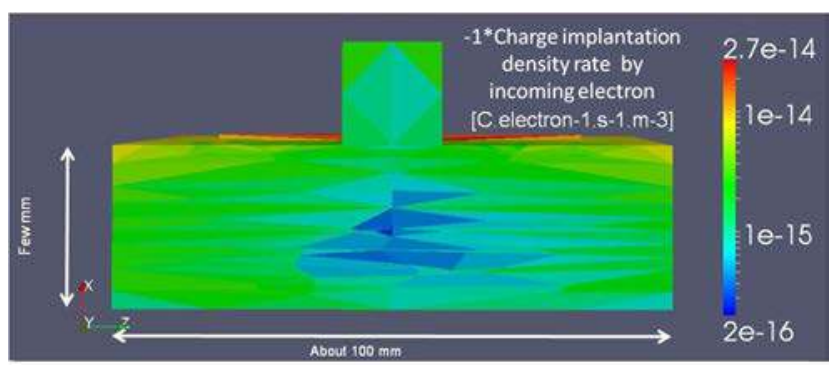

Fig. 12. Clipping of the charge implantation density rate by incoming electron computed by GRAS. View scaled along the thickness direction.

of the card and no major anisotropy is observed. The particle charge is added in the whole tetrahedron where the particle stops. The charge deposition rate computed by Geant 4 models the electrons deposited and removed by the space environment. The IC simulation, with SPIS IC, will use this 3-D scoring data to compute the movement of low energy charges by diffusionconvection [7].

\section{InTERnAl ChARging StUdy}

\section{A. Modeling Choice}

On the basis of the previous results, an IC analysis has been performed with SPIS-IC, presented in Section II-B, and considering the present scoring mesh as a computational domain for the charge transport in the matter models.

Material properties and assignment are summarized in Table V where RCC is the radiation-induced conductivity
TABLE V

IC Material Properties

\begin{tabular}{|l|l|c|c|c|}
\multicolumn{5}{|c|}{ Internal charging materials } \\
\hline Components & material name & RCC $\left[\mathrm{ohm}^{-1} \cdot \mathrm{m}^{-1}\right]$ & BUC [ohm-1.m-1] & RCP [-] \\
\hline High voltage card & FR4 & $1.73 \mathrm{E}-20$ & $8.48 \mathrm{E}-16$ & 1.07 \\
\hline Botom surface & pure conductor & & & \\
\hline Element A & pure conductor & & & \\
\hline Element B & coverglass & $1.0 \mathrm{E}-13$ & $1.0 \mathrm{E}-13$ & 1 \\
\hline Element C & pure conductor & & & \\
\hline
\end{tabular}

coefficient, RCP is the power factor, and BUC is the dark conductivity used in SPIS IC [22]. In this paper, to model a worst-case scenario from the charging perspective, the component B must be a dielectric such as a coverglass material, which models the glass used to protect the components on the high-voltage card. Coverglass is compound by silicon, also used as material for several electrical circuit elements. The body of the high-voltage card is considered as epoxy with the FR4 "Flame-Retardant 4" material, except the largest surface opposite the components, which is a pure conductor. Finally, the FPGA and the copper strip on the high-voltage card are considered pure conductors. All components are electrically grounded.

As explained in Section III-B, the space environment used for the radiation transport simulation is electron fluence corresponding to an orbit of three years for the Jason-3 mission. The majority of the fluence received on a PEO of three years mostly occurs over a short time period when the spacecraft crosses the polar regions. For SPIS-IC simulations, four different cases have been considered: the total fluence is received in one day, one week, one month, and one year. In this paper, the results are similar in all these cases in the sense that the same maximum electric potentials and electric fields are reached for the other considered duration. By the way, when the considered duration is smaller, the maximum electric potential is reached in a smaller time. This scalability is due to the fact that the electric potentials and electric field are low. Thus, the conduction is not important in this simulation. The only important phenomenon is the charge implantation map. In that case, the electric potential and the electric field are only dependent on the total accumulated charge and not on the accumulation charge rate. Therefore, only the one-day duration result is presented as the worst-case scenario where the maximum electric field and electric potential are reached quite fast.

\section{B. Results}

Figs. 13 and 14 show the results of the IC during one day. In Fig. 13, the curves of the electric field seem linear but if the simulation continues to run, a stationary electric field would be reached after a very long time. In this paper, we considered that the main part of the three-year fluence occurs over one day without relaxation period. In reality, the space environment and then the dose rate received during this period is variable so the electric field is never stationary. This should be analyzed in another study using both refined definition of the environment varying in time and improved capabilities of SPIS-IC [25]. The high-voltage card is the component with the highest value of 


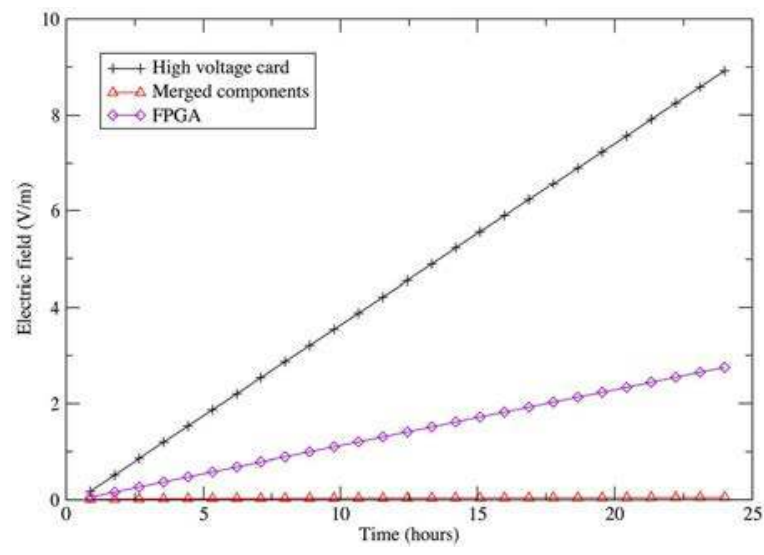

Fig. 13. Electric field versus time for the one-day duration for each component.

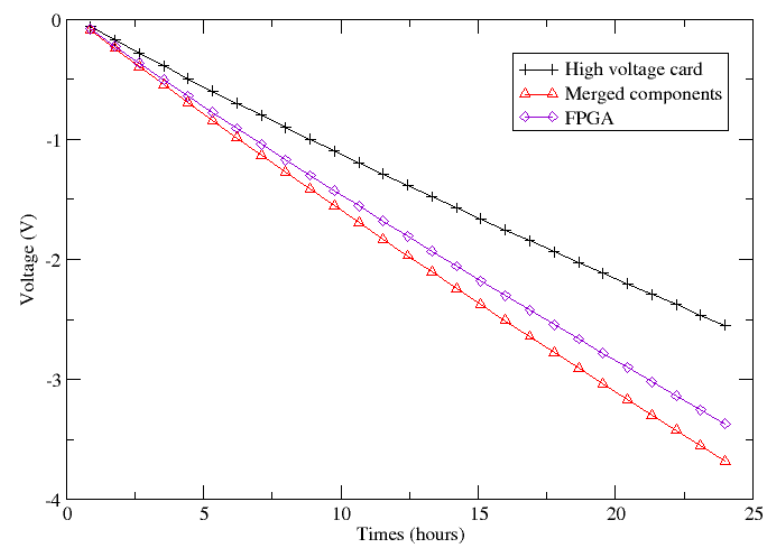

Fig. 14. Maximal electric potential versus time for one day.

the maximum electrical field, which is about $9 \mathrm{~V} / \mathrm{m}$. The exact threshold, above which arcing and discharge occur, depends on numerous factors and can be difficult to determine without precise (and often destructive) experimental measurements. However, we can say that these low values of the electric field are not sufficient to create a discharge.

It is tempting to compare these results with ones given by other IC models, like DICTAT, usually used for first quick calculations. Based on analytical models, DICTAT may consider two simple types of geometries, with various possible grounding schemes. The shielding parameter is an aluminum thickness. The sector shielding analysis results show that it might be difficult to consider only one thickness value for realistic systems. Moreover, DICTAT simulation results have shown that the final maximum potential can change of several orders of magnitude with the AMBER values of shielding thickness used. Last, for this study environment, a similar geometry, models implemented in DICTAT are not able to give precise results for a shielding above $4 \mathrm{~mm}$ of aluminum. For these reasons, results from DICTAT, and more generally 1-D models in cases of complex geometries, like AMBER, should be taken with care. Extrapolating results computed by DICTAT, we still obtain a maximum dielectric surface voltage of $2,5 \mathrm{~V}$ for an averaged shielding of about $11 \mathrm{~mm}$, which seems consistent with results from this paper.
In Fig. 14, SPIS IC considers all tetrahedra for each group (high-voltage card, merged components, or FPGA), and for each time step, the maximum of the electric potential of these tetrahedra is plotted as a y-coordinate. As illustrated in Fig. 14, the final electric potential of modeled components on the card remains in a maximal electric potential range about $-4 \mathrm{~V}$ after the one day of the equivalent worst-case scenario.

With values lower than $10 \mathrm{~V} / \mathrm{m}$ for the electrical field and lower than $4 \mathrm{~V}$ for the maximal electric potential, no electrical breakdown should appear. In this analysis, these values are low so it is not necessary to display them in 3-D.

One should take care to avoid generalizing these results by considering two aspects. First, the primary induced dose and charge rates deeply depend on the orbit definition and the variations of the space environment. On other orbits, trapped particles may promptly induce strong dose rate and charge rate in a short time and in an anisotropic way. It seems relevant and prudent to perform deeper IC analysis for each new mission profile and/or space event. Moreover, the spacecraft hub has not been considered in this section. Depending on particle energies, modeling the hub can increase the IC risks. The impact of the spacecraft hub is analyzed in Section V. Finally, this paper outlines that the shielding is able to decrease the electron implantation in the material. In the case of this paper, the only important phenomenon is the implantation. The conduction is negligible. Thus, the shielding is able to mitigate the risk of electrical breakdowns for components inside the shielding. When the conductivity or the radiations induce conductivity plays a more important role, as for example for higher intensity or for higher irradiation time, the shielding may also decrease the conductivity. Thus, it would lead to increase in the risk of discharge.

\section{IMPACT OF THE JASON3 SPACECRAFT BODY}

By its mass and extension, the Jason-3 spacecraft constitutes an additional shielding due to the location of Amber on the spacecraft. This additional shielding presents a strong anisotropy. It is important to be able to model the body of Jason-3 and evaluate its impact. This anisotropy shielding due to the Jason-3 body may lead to a severe anisotropy regarding the charge deposition rate in the high-voltage card and then the risk of IC can change. The dimension of the Jason- 3 body is about $1 \mathrm{~m} \times 1 \mathrm{~m} \times 2.2 \mathrm{~m}$ and its mass is approximately $500 \mathrm{~kg}$ [31]. Modeling the full body of Jason-3 and the Amber instrument in the same simulation is difficult because the dimensions of both systems are very different, about $2.2 \mathrm{~m}^{3}$ for Jason-3 and about $4.10^{-2} \mathrm{~m}^{3}$ for the Amber instrument. The source to model the space environment used in the GRAS/Geant4 must surround the whole Jason/Amber system. To obtain results with a similar and sufficient statistic, the number of events must be drastically superior and the simulation time greater than seven years, which is unacceptable if neither simplification nor proper biasing is used. To address this issue, a sector shielding analysis done with SSAM has been used to evaluate the equivalent shielding seen by Amber considering the Jason-3 body, modeled as an equivalent hollow box. On this basis, an equivalent aluminum plate has been 


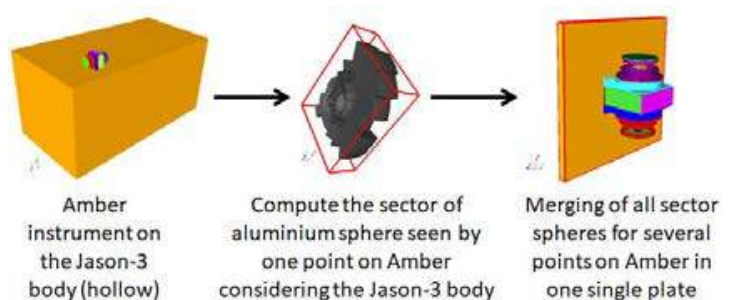

Fig. 15. Simplification of Jason-3 hub to address the multiscale modeling.

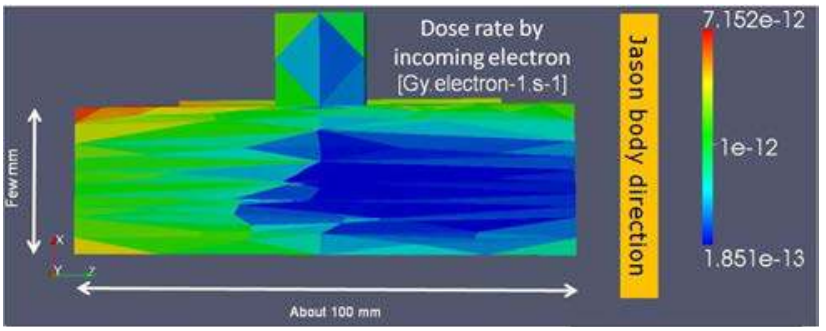

Fig. 16. Clipping of the dose rate over the high-voltage card with the Jason-3 hub. View scaled along the thickness view.

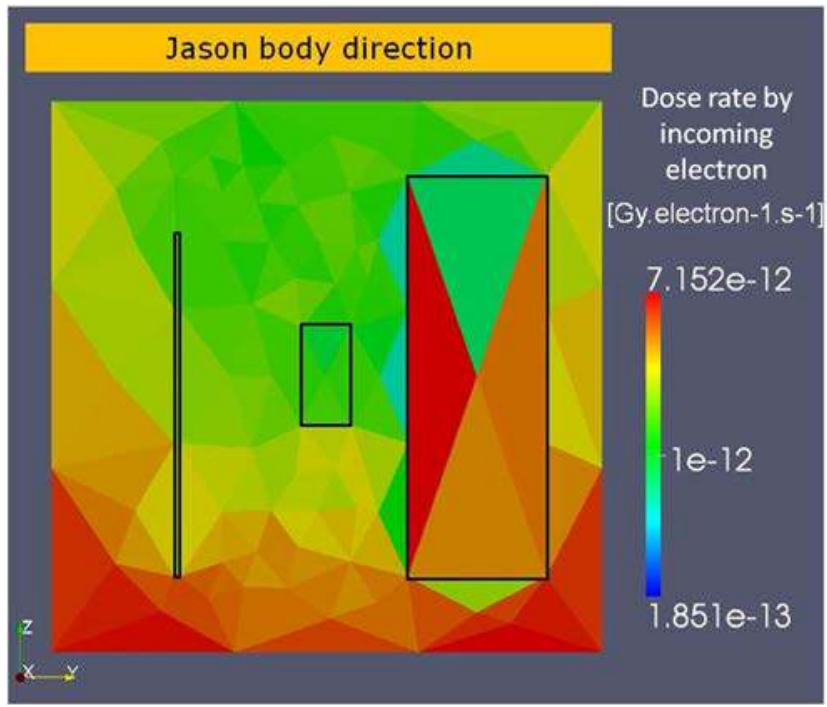

Fig. 17. Top view of the scoring of the dose rate over high-voltage card considering the influence of the Jason-3 body.

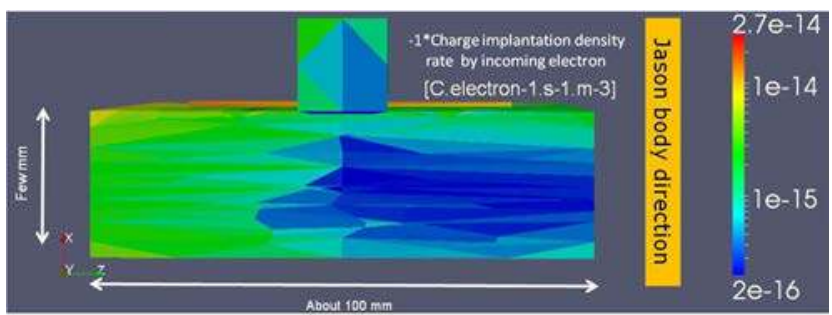

Fig. 18. Clipping of the absolute charge deposition rate density with the Jason-3 hub. View scaled along the thickness view.

defined with a thickness of $2 \mathrm{~cm}$. Considering the retained isotropic space environment, electrons do not have enough energy to cross such thickness; thus, no electron is able to cross the Jason-3 body. This approach is presented in Fig. 15.

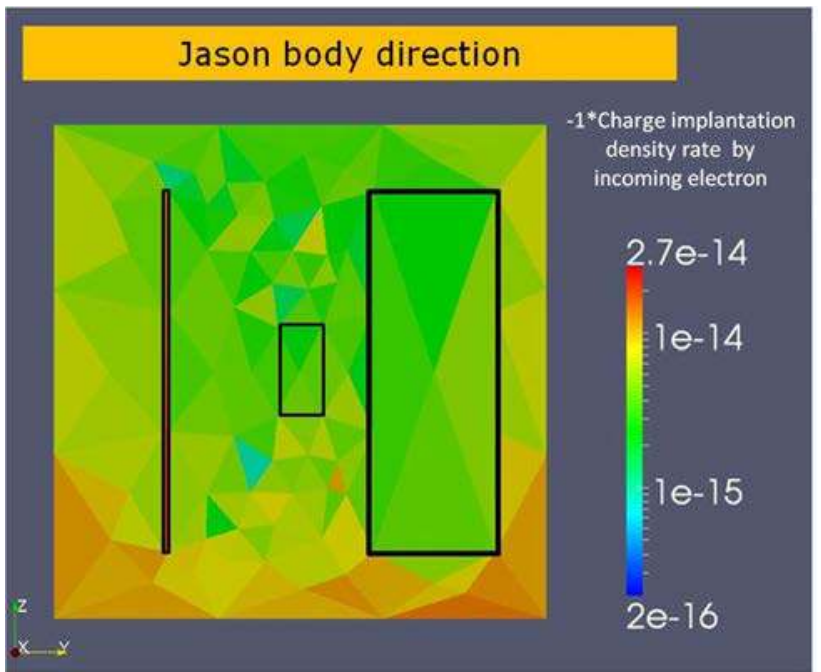

Fig. 19. Top view of the scoring of absolute charge deposition rate density over the high-voltage card considering the influence of the Jason- 3 body

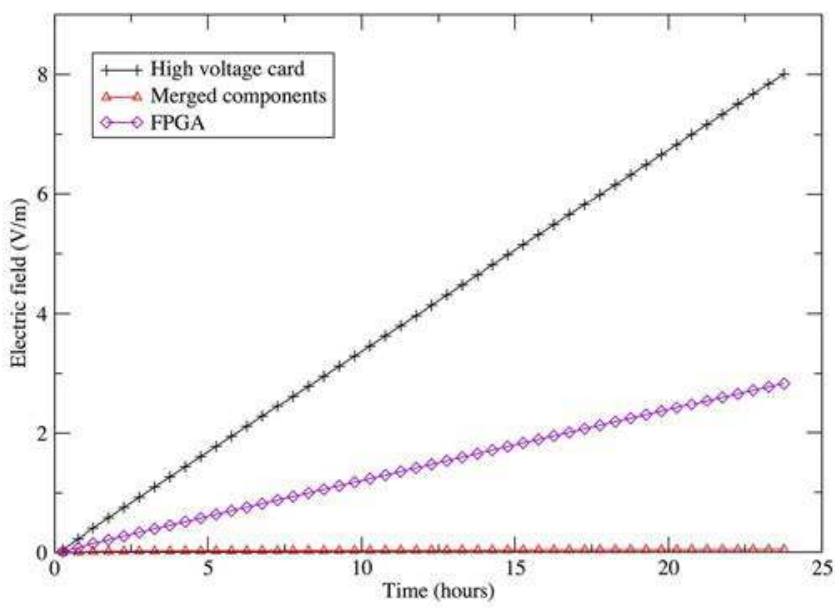

Fig. 20. Results for a one-day duration, the maximum electric field versus the time for each group of components considering the influence of the Jason-3.

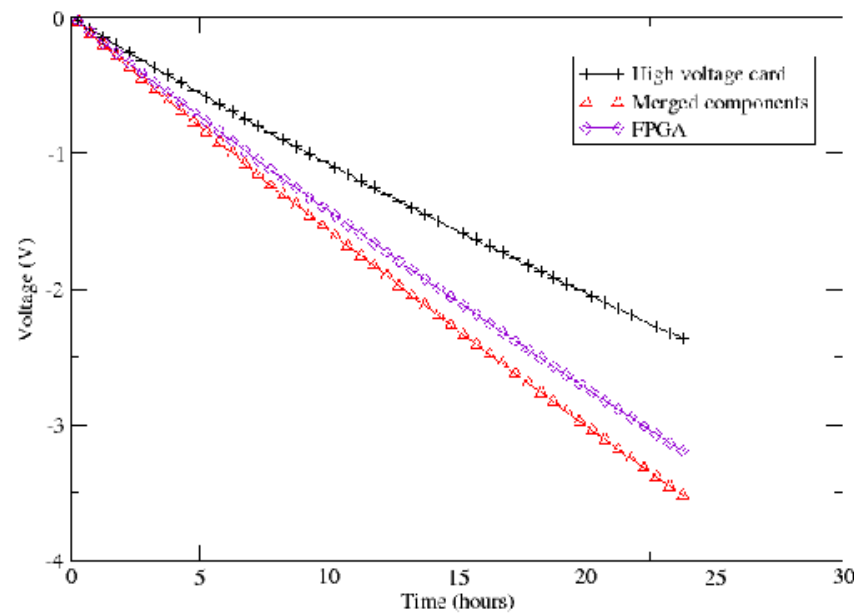

Fig. 21. Results for a one-day duration, the maximum electric potential versus the time considering the influence of the Jason-3 body.

The modeling chain to compute the IC in the high-voltage card of AMBER has been reused on this new geometry in 


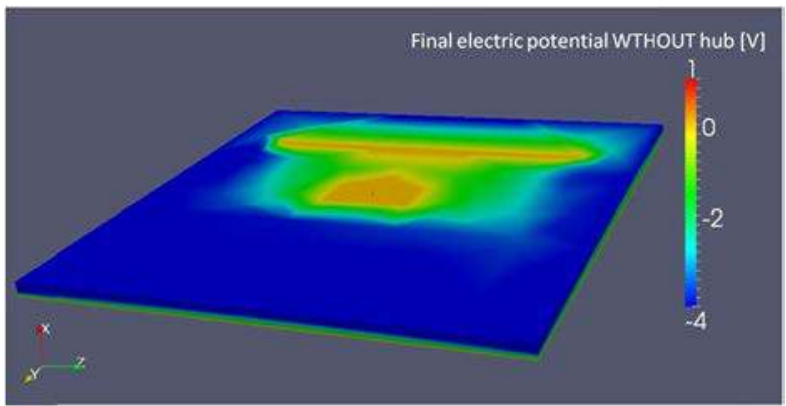

Fig. 22. Results for one-day duration, final electric potential of the highvoltage card, simulation without the Jason-3 body.

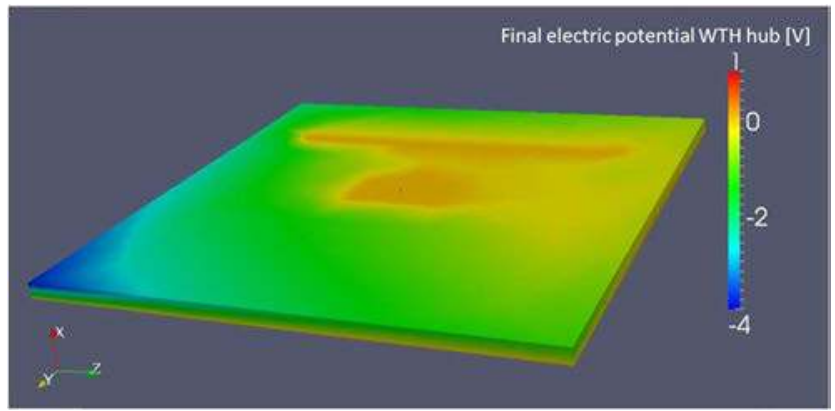

Fig. 23. Results for the one-day duration, final electric potential of the high-voltage card, simulation with the Jason-3 body.

the same way with the same electron flux. The results of the radiation transport analysis are presented in Figs. 16-19. The Jason-3 CAD model has an important influence on Geant4/GRAS results. Both the dose rate and charge deposition rate are lower on the part of the card near the Jason-3 body $(+z$ - direction compared to the high-voltage card).

The results of the IC are presented in Figs. 20 and 21. The trend of the plots and the maximum values for the electrical field and the electric potential are the same compared to the modeling without the Jason-3 body. In addition, the 3-D results of the electric potential show the IC results are not exactly the same as illustrated in Figs. 22 and 23. The gradient of the electric potential is still maximum along the thickness of the high-voltage card but the Jason-3 body creates a big anisotropy concerning where this maximum gradient is. As shown in this paper, the spacecraft may create anisotropy for the dose rate and the charge deposition rate. Besides, considering other space environments, like where the energy of electrons is higher, the spacecraft can also slow particles, such as electrons, enough that the dose rate and charge deposition rate may be higher, increasing the IC risk.

\section{CONCLUSION}

The modeling chain based on EDGE, MoORa/GRAS, and SPIS-IC has been presented and used to model the IC of the high-voltage card in the AMBER instrument onboard Jason-3. The results confirm that the risk of electrical break due to IC is low for AMBER onboard the Jason-3 mission, which is fully consistent because no IC related anomalies have been identified so far on AMBER. The EDGE/MoOra/GRAS/SPIS-IC modeling chain has fully confirmed its capability to model finely, and in an engineering context, realistic systems and scientific payloads. The comparison of results against previous works and other simulation codes also confirm the confidence in the implemented numerical models (e.g., SSAM, GRAS, SPIS-IC). Thanks to the adaptability of this chain, new AMBER geometry designs, orbit profiles, components, and materials could be easily modeled; ascertaining optimum configurations to prevent IC risks. This paper presented in detail a step-by-step methodology to model the IC effects of a space instrument realized with a dedicated modeling chain.

The application of the EDGE/MoOra/GRAS/SPIS-IC modeling chain is not limited to IC studies. The possibility to easily compute cumulative dose, using reference Monte Carlo models and through a modern easy-to-use GUI, greatly simplifies simulations for radiation transport readiness analyses of space systems. With less than $10 \mathrm{kRad}$ in the present case, thanks to a proper shielding design, one may notice that the TID computed over the high-voltage components during three years, is lower than the critical radiation-induced degradation threshold for numerous computer cards, including for very low-cost and nonspace-ready ARM-based cards [32], [33] which opens perspectives for the use of nonspace designed components on future scientific instruments.

\section{REFERENCES}

[1] P. Sarrailh, "Internal charging simulation at a Galileo like orbit-Effect of the anisotropic shielding and of the environment definition," in Proc. 12th Geant4 Space Users Workshop, Apr. 2017, pp. 1-12.

[2] D. Payan, "AMBER, the French plasma monitor on-board JASON3," in Proc. 15th Spacecraft Charging Technol. Conf., Kobe, Japan, Jun. 2018, pp. $1-18$.

[3] J. A. Sauvaud et al., "AMBER: The French plasma detector aboard JASON3-First results," in Proc. SPACECOM, Dec. 2017, p. 1.

[4] JASON-3-Satellite Missions-eoPortal Directory. Accessed: Apr. 29, 2019. [Online]. Available: https://directory.eoportal.org/web/ eoportal/satellite-missions/j/jason-3

[5] D. J. Rodgers, K. A. Ryden, G. L. Wrenn, P. M. Latham, J. Sorensen, and L. Levy, "An engineering tool for the prediction of internal dielectric charging," in Proc. 6th Spacecraft Charging Technol. Conf., Hanscom AFB, MA, USA, Nov. 1998, pp. 1-3.

[6] W. Kim, I. Jun, and H. B. Garrett, "NUMIT 2.0: The latest version of the JPL internal charging analysis code," in Proc. 12th Spacecraft Charging Technol. Conf., Kitakyushu, Japan, May 2012, pp. 1-3.

[7] P. Sarailh et al., "Validation through experiments of a 3-D time-dependent model of internal charging," IEEE Trans. Plasma Sci., vol. 45, no. 9, pp. 2566-2572, Sep. 2017.

[8] S. Ibarmia et al., "Geant4 tools and interfaces from ELSHIELD project," in Proc. 9th Geant4 Space User's Workshop, Barcelona, Spain, Mar. 2013, p. 1.

[9] V. N. Ivanchenko, "Validation results from ELSHIELD project," in Proc. 16th Geant4 Workshop, Sep. 2011, p. 1.

[10] F. Lei et al., "Collaborative iterative radiation shielding optimisation system," in Proc. 12th Geant4 Space User's Workshop, Apr. 2017, p. 1.

[11] W. Kim, J. Z. Chinn, I. Katz, H. B. Garrett, and K. F. Wong, "3-D NUMIT: A general 3-D internal charging code," IEEE Trans. Plasma Sci., vol. 45, no. 8, pp. 2298-2302, Aug. 2017.

[12] Geometry Description Markup Language (GDML). Accessed: Apr. 29, 2019. [Online]. Available: https://gdml.web.cern.ch/GDML/

[13] R. Chytracek, J. McCormick, W. Pokorski, and G. Santin, "Geometry description markup language for physics simulation and analysis applications," IEEE Trans. Nucl. Sci., vol. 53, no. 5, pp. 2892-2896, Oct. 2006

[14] EDGE-SpaceSuite. Accessed: Apr. 29, 2019. [Online]. Available: http://www.space-suite.com/

[15] G. Santin, "Radiation effects on sensors and technologies for JUICE," in Proc. JUICE Instrum. Workshop, Nov. 2011, pp. 1-54.

[16] Overview | Geant4. Accessed: Apr. 29, 2019. [Online]. Available: http://geant4.web.cern.ch/ 
[17] TEC-EPS-Geant4 Radiation Analysis for Space. Accessed: Apr. 29, 2019. [Online]. Available: http://space-env.esa.int/index.php/geant4radiation-analysis-for-714space.html

[18] G. Santin, V. Ivanchenko, H. Evans, P. Nieminen, and E. Daly, "GRAS: A general-purpose 3-D modular simulation tool for space environment effects analysis," IEEE Trans. Nucl. Sci., vol. 52, no. 6, pp. 2294-2299, Dec. 2005

[19] MoORa-SpaceSuite. Accessed: Apr. 29, 2019. [Online]. Available: http://www.space-suite.com/

[20] J.-F. Roussel et al., "SPIS open-source code: Methods, capabilities, achievements, and prospects," IEEE Trans. Plasma Sci., vol. 36, no. 5, pp. 2360-2368, Oct. 2008.

[21] B. Thiebault et al., "SPIS 5.1: An innovative approach for spacecraft plasma modeling," IEEE Trans. Plasma Sci., vol. 43, no. 9, pp. 2782-2788, Sep. 2015

[22] Spacecraft Plasma Interaction Network in Europe-SPINE Home. Accessed: Apr. 29, 2019. [Online]. Available: http://www.spis.org/

[23] G. Hubert et al., "Towards a multi-scale platform to investigate multiconstraints and effects induced by space radiation environments on system boards," in Proc. 68th Int. Astron. Congr., 2017.

[24] EREMS et. al., "Flux d'électrons et de protons GEO et JASON2 Réf. PiresCas Ambre.xls," CNES Internal Rep., Tech. Note.

[25] P. Sarrailh et al., "Simulation of the effect of time-dependent electron environment on the internal charging dynamic using SPIS-IC," in Proc. 15th Spacecraft Charging Technol. Conf., Kobe, Japan, Jun. 2018, p. 1.

[26] G. Santin, "Normalisation modelling sources," Geant4 Tutorial, Paris, France, Jun. 2007.

[27] N. Sukhaseum et al., "Analyse en dose et calcul de flux transporté pour l'équipement AMBRE," TRAD, CNES Internal Rep., Tech. Rep. TRAD/ERE/JAS/AMB/PFP/150911, Rev. 1, Toulouse, France, Oct. 2011.
[28] V. Ivanchenko. Electromagnetic Physics Standard Category. Geant4 Release. Accessed: Apr. 29, 2019. [Online]. Available: http://geant4.in2p3.fr/2013/resources/L10-EM742

[29] D. Wright. A Short Guide to Choosing Physics Lists. Geant4 Release. Accessed: Apr. 29, 2019. [Online]. Available: http://geant4.in2p3.fr/IMG/pdf_PhysicsLists.pdf

[30] TEC-EPS-Internal Charging Tool-DICTAT. Accessed: Apr. 29, 2019. [Online]. Available: http://space-env.esa.int/index.php/dictat.html

[31] The NOAA National Environmental Satellite, Data, and Information Service (NESDIS). (Sep. 2018). Jason-3 Satellite-Spacecraft. [Online]. Available: https://www.nesdis.noaa.gov/jason-3/spacecraft.html

[32] D. P. Violette, "Arduino-raspberry Pi: Hobbyist hardware and radiation total dose degradation," Student Pathway Intern NASA Goddard Space Flight Center, Univ. Connecticut, Greenbelt, MD, USA, Tech. Rep., Sep. 2014.

[33] B. Jeanty-Ruard et al., "New integrated pre-processing chain for radiations and internal charging analysis to model time variations impacts," in Proc. 14th Eur. Space Weather Week, Ostend, Belgium, Dec. 2017, p. 1. [Online]. Available: http://www.stce.be/esww14/contributions/ public//S8-P1/S8-P1-04-CHAMPLAINAmandine/PosterESWW2017 poster_V1.0.pdf 